\title{
Germanica
}

GERMANICA

$41 \mid 2007$

Le livret d'opéra en langue allemande au $\mathrm{XX} \mathrm{X}^{\mathrm{e}}$ siècle : ruptures et reprises

\section{Leonce und Lena sur la scène lyrique est-allemande au tournant des années 1970-80}

Leonce und Lena auf der Opernbühne der DDR um 1980

\section{Laetitia Devos}

\section{OpenEdition}

\section{Journals}

Édition électronique

URL : http://journals.openedition.org/germanica/482

DOI : $10.4000 /$ germanica.482

ISSN : 2107-0784

Éditeur

Université de Lille

Édition imprimée

Date de publication : 1 décembre 2007

Pagination : 113-129

ISBN : 978-2-913857-20-9

ISSN : 0984-2632

Référence électronique

Laetitia Devos, « Leonce und Lena sur la scène lyrique est-allemande au tournant des années

1970-80 », Germanica [En ligne], 41 | 2007, mis en ligne le 01 décembre 2009, consulté le 06 octobre

2020. URL : http://journals.openedition.org/germanica/482 ; DOI : https://doi.org/10.4000/germanica. 482

Ce document a été généré automatiquement le 6 octobre 2020.

(c) Tous droits réservés 


\title{
Leonce und Lena sur la scène lyrique est-allemande au tournant des années 1970-80
}

\author{
Leonce und Lena auf der Opernbühne der DDR um 1980
}

\author{
Laetitia Devos
}

1 L'opéra Wozzeck d'Alban Berg (1925) marqua l'histoire de l'opéra au vingtième siècle et fut le premier d'une longue série d'opéras d'après les pièces de Georg Büchner (1813-1837) : le vingtième siècle n'en connut pas moins de quinze, dont treize en Allemagne.

2 Büchner fut aussi célébré par de grands auteurs communistes, comme Anna Seghers qui fut l'une des premières après la Deuxième Guerre mondiale à recevoir le Prix Büchner (en 1947), ou encore Georg Lukács qui, dès 1937, en exil à Moscou, publiait un article pour rétablir « le Büchner authentique » qu'il opposait au Büchner «falsifié par l'idéologie nazie $»^{1}$.

$3 \mathrm{Au}$ vu de la référence que constituait Büchner tant dans l'histoire de l'opéra du vingtième siècle que dans l'histoire des idées marxistes, on pourrait s'attendre à voir l'œuvre de Büchner figurer au "palmarès » des opéras joués en RDA. Or, ce fut loin d'être le cas : Wozzeck de Berg fut joué seulement trois fois en quarante ans (1949-89) au Staatsoper de Berlin ${ }^{2}$; quant aux créations d'opéras d'après Büchner en RDA, elles furent au nombre de cinq. Il est intéressant de relever que trois de ces créations furent strictement contemporaines et composées au tournant des années 1970-80. Il s'agit de Leonce und Lena de Paul Dessau créé en novembre 1979 au Staatsoper de Berlin; Leonce und Lena de Thomas Hertel achevé dès 1979 et créé en 1981 à Greifswald; Büchner de Friedrich Schenker composé de 1978 à 1981 et créé en 1987 au Staatsoper (Apollosaal) de Berlin.

4 Composer plusieurs opéras d'après le même auteur dans le petit pays qu'était la RDA pourrait ne pas relever d'une simple coïncidence. Nous nous intéresserons ici de plus près au Leonce und Lena de Paul Dessau ${ }^{3}$ qui marqua le début de la « série Büchner » à 
l'opéra dans les années 1980 et qui fut par ailleurs le plus connu des cinq. Nous le mettrons en regard avec l'opéra du même nom de Thomas Hertel ${ }^{4}$, beaucoup moins connu, mais composé exactement simultanément et, si l'on en croit Thomas Hertel, tout à fait indépendamment et sans aucune connaissance de celui de Dessau: Hertel, novice qu'il était à l'époque, n'aurait en effet (à ses propres dires ${ }^{5}$ et selon toute vraisemblance) jamais osé soutenir la comparaison avec un compositeur aussi renommé que Dessau s'il avait su que ce dernier travaillait lui aussi à un Leonce und Lena.

5 La concomitance de ces deux opéras homonymes ne relevait donc ni de la concertation, ni du plagiat, ni d'une influence directe d'un compositeur sur l'autre. Si l'on se place dans une perspective bourdieusienne, il semble incontournable de s'intéresser aux " points de repère, phares ou repoussoirs, par rapport auxquels se [sont] défini[s] le[s] projet[s] créateur[s] $»^{6}$. La stricte contemporanéité de deux opéras d'après Büchner s'explique en effet sans doute par l'actualité de l'écrivain du Vormärz dans la RDA de l'époque. Il ne s'agit cependant pas ici « d'anéantir le créateur par la reconstruction de l'univers des déterminations sociales qui s'exercent sur lui, et de réduire l'œuvre au pur produit d'un milieu» mais «de décrire et de comprendre le travail spécifique que l'écrivain [et le compositeur] a dû accomplir, à la fois contre ces déterminations, et grâce à elles, pour se produire comme créateur, c'est-à-dire comme sujet de sa propre création $»^{7}$.

6 Comme l'avait fait remarquer Boris Blacher à Gottfried von Einem en $1948^{8}$, transposer la comédie Leonce und Lena en livret d'opéra était un véritable défi car cette comédie hybride était parsemée de jeux de mots, de références philosophiques (satire de l'idéalisme), de citations authentiques ou parodiques (tirées principalement des œuvres de Musset, Shakespeare, Lenz, Tieck et Brentano). Les mots y avaient la prépondérance sur l'action. Aussi le passage au livret d'opéra dont on sait qu'il implique une réduction du texte, qui, de plus, une fois chanté, perd parfois en compréhensibilité, ne risquait-il pas de se faire aux dépens des jeux de mots et traits d'esprits büchnériens, et par là même, aux dépens de tout ce qui faisait l'intérêt de la pièce?

7 Nous verrons comment Dessau et Hertel s'efforcèrent chacun à leur manière de retranscrire le persiflage sur lequel reposait la comédie de Büchner et quelle signification particulière la subversion de l'idéal classique prenait dans le contexte de la RDA.

\section{Circonstances de création}

8 La caractéristique principale de la politique culturelle communiste était d'accorder un grand crédit au rôle qu'avaient à jouer les artistes dans la construction de la société communiste. L'opéra, en raison de la possibilité qu'il offrait de présenter une action exemplaire tout en l'illustrant musicalement, fut l'objet de nombreux débats et attentes en RDA ${ }^{9}$. Si les années 1950-60 furent, à quelques exceptions près ${ }^{10}$, celles de la création d'opéras néo-classiques présentant des conflits de classe manichéens doublés d'un dualisme musical opposant accords consonants et dissonants ${ }^{11}$, cette tendance commença à cohabiter au milieu des années 1960 avec des opéras à la «tonalité élargie » frisant un sérialisme prudent ${ }^{12}$ (car proscrit). En 1968, le $9^{\mathrm{e}}$ plenum du Comité Central du SED vint interrompre cette libéralisation en rappelant sur un ton menaçant les dangers du «modernisme» qui était «la porte ouverte aux tendances contre- 
révolutionnaires ». Mais l'arrivée de Honecker au pouvoir et la promesse d'abolition des « tabous » en art relancèrent l'ouverture progressive de la musique à la modernité 13 .

De ce point de vue, Paul Dessau (1894-1979) fut l'un des précurseurs : il entreprit, selon sa propre expression, un travail d'« éducation des princes » et ce malgré les difficultés des débuts (Die Verurteilung des Lukullus en 1951 sur un livret de Brecht fut la cible de la lutte contre le "formalisme», c'est-à-dire contre toute forme de modernité). Exilé pendant la guerre aux États-Unis, où il côtoya Brecht et Schoenberg, Paul Dessau s'installa en RDA en 1949, adhéra au SED et prit la nationalité est-allemande ${ }^{14}$. S'il fut rapidement considéré par les autorités comme un compositeur «national», il n'en préserva pas moins un style musical moderne, sa singularité et des opinions personnelles voire critiques. La circonspection dont il fit preuve est visible au travers de son parcours institutionnel : alors qu'il reçut des prix nationaux à quatre reprises, il n'occupa jamais de fonction officielle importante. La prudence de son engagement idéologique est encore vérifiable peu avant la composition de Leonce und Lena: dans l'opéra qui précède immédiatement Leonce und Lena, Einstein (1974), Dessau hésita quant à la fin à donner à cet opéra, comme si pour lui le communisme n'était plus une réponse à certains problèmes (le danger nucléaire). Toutefois, deux ans plus tard, dans "l'affaire Biermann ", Dessau, contrairement à de nombreux artistes, se rangea du côté du Parti, sans doute par esprit de discipline. À la fin de sa vie, Paul Dessau restait donc malgré tout un communiste convaincu.

Dessau mourut en juin 1979, cinq mois avant la création de son dernier opéra Leonce und Lena, prévue pour son $85^{\mathrm{e}}$ anniversaire. La programmation de cet opéra avait rencontré des difficultés, car les autorités culturelles trouvaient que l'œuvre manquait de teneur politico-sociale ${ }^{15}$ et sans doute le parallèle entre la sclérose des Royaumes «Popo » et «Pipi » et celle de la RDA ne leur échappa-t-il pas ${ }^{16}$. Il fut prétexté que le salaire demandé par la chanteuse qu'avait choisie Dessau était bien trop élevé. Dessau crut même un temps devoir renoncer à la création ${ }^{17}$. Ces ultimes obstacles affectèrent beaucoup Dessau, alors âgé de 84 ans, lui qui avait œuvré sans relâche depuis trente années pour la musique en RDA. Création finalement posthume, Leonce und Lena fut mis en scène par Ruth Berghaus (épouse de Dessau) en novembre 1979 au Staatsoper de Berlin $^{18}$.

L'autre compositeur dont il sera question ici, Thomas Hertel, était moins célèbre, mais son nom était loin d'être inconnu des milieux musicaux: né en 1951 en Thuringe, Hertel fit des études de musicologie à l'Université de Halle. En 1973, il fut admis dans la classe de Siegfried Matthus à l'Akademie der Künste. Dès 1974, il fut responsable de la musique de scène au Staatstheater de Dresde. Sans jamais adhérer au SED, il dirigea la section «jeunes compositeurs» de l'Union des Compositeurs de Dresde et reçut plusieurs Prix de composition. En dehors de la musique écrite pour le théatre, Hertel composa deux opéras (Leonce und Lena puis Hölderlin-Report) et chérissait la collaboration avec les autres branches artistiques comme les arts plastiques, créant des œuvres d'art plurimédiales ${ }^{19}$. Il rencontra cependant des obstacles à sa liberté artistique : il n'eut par exemple jamais l'autorisation de composer un opéra d'après Heiner Müller. L'opéra Leonce und Lena était une commande du Staatstheater de Dresde où il travaillait. Alors que Hertel avait déjà achevé la partition, l'avait présentée publiquement au piano et que l'œuvre était programmée, le Staatstheater de Dresde connut un brusque changement de direction et le nouveau directeur refusa de jouer du 
Büchner dans son établissement. Cependant, grâce à un jeune metteur en scène qui avait entendu parler de l'opéra et souhaitait le monter, Leonce und Lena put être finalement joué au théâtre de Greifswald. Là, tout ne se passa pas sans heurts non plus : le metteur en scène rencontra des problèmes avec les chanteurs et avec la direction du théâtre et dut arrêter le projet. Le directeur du théâtre termina de monter l'opéra luimême en quelques jours. En 1985, Hertel obtint un visa lui permettant de quitter la RDA et continua à se consacrer à des projets musicaux et plurimédiaux à l'Ouest (Munich, Vienne et Strasbourg). Depuis 2002, il est responsable de la musique au théâtre de Leipzig.

Malgré tout ce qui éloigne Hertel et Dessau, tous deux s'emparèrent de Büchner à un moment-clef de leur carrière : premier opéra pour l'un et dernier pour l'autre - mais une fin pour tous les deux puisque Hertel envisageait de quitter la RDA et que Dessau prévoyait sa fin prochaine ${ }^{20}$. Le librettiste de Dessau, Thomas Körner, attendait pour sa part d'obtenir un visa pour quitter le pays. La comédie Leonce und Lena était-elle donc une manière de prendre congé de la RDA ?

On constate qu'au tournant des années 1970-80, les compositeurs d'opéras à la pointe de l'avant-garde en RDA recouraient volontiers à des modèles littéraires du passé pour leurs livrets. Parmi les écrivains choisis, on trouve, outre Büchner, Lenz (R. Hot, die Hitze de Goldmann en 1977), Kafka (Die Verwandlung de Dittrich en 1983) et Voltaire (Candide de Bredemeyer en 1981, créé en 1987) - des écrivains certes fort différents, mais dont le point commun était de porter un regard très critique sur la société de leur époque. Ces auteurs avaient été plus ou moins « censurés » jusqu'au début des années 1970 en RDA. Que dire du choix plus particulier de Büchner «au sein de l'univers des choix compossibles » (Bourdieu) de livrets d'opéra? Hertel explique que Büchner était d'une grande actualité tout en étant un écrivain du début du $\mathrm{XIX}^{\mathrm{e}}$ siècle, si bien qu'il était beaucoup moins périlleux politiquement d'écrire un opéra d'après Büchner que d'après un contemporain comme Heiner Müller (comme il aurait souhaité le faire) ${ }^{21}$. Quant à Dessau, on peut relever que c'est la première et unique fois qu'il choisit une source littéraire datant d'un siècle passé pour un de ses opéras. Tout comme Hertel, il savait qu'un opéra d'après Leonce und Lena avait déjà été composé en RDA, presque vingt ans plus tôt, par Kurt Schwaen. Mais ni Dessau ni Hertel ne voyaient dans cet opéra de Schwaen un obstacle à la réalisation d'un opéra du même titre, car ils concevaient Leonce und Lena tout à fait différemment de leur prédécesseur ${ }^{22}$.

L'opéra de Kurt Schwaen, créé à l'automne 1961 au Staatsoper de Berlin, venait justement d'être repris en 1977 à Greifswald dans une mise en scène de Dieter Reuscher qui modifiait considérablement l'opéra néo-classique à numéros de Schwaen. Alors que Schwaen présentait Leonce und Lena comme une histoire d'amour inoffensive, Reuscher ajouta des scènes qui donnaient une perspective sociopolitique marxiste à l'opéra (opposition entre les pauvres paysans d'un côté et le couple royal de l'autre). Cette mise en scène de la fin des années 1970 illustrait le dogmatisme persistant de certains. Dans les deux premières décennies de la RDA, Büchner avait été soumis à une déformation qui le présentait en précurseur indéniable de Marx. Mais paradoxalement, Büchner n'avait quasiment jamais été joué jusqu'au début des années 1970 en RDA, sans doute en raison de l'ambiguïté de ses œuvres ${ }^{23}$.

Avec « l'affaire Biermann » et l'indignation de nombreux intellectuels, Büchner devint de plus en plus actuel, comme le relève Daniela Reinhold : 
Une partie de la jeune génération se reconnut dans les biographies d'intellectuels en marge de la société comme Bettina von Arnim, Georg Büchner, Karoline von Günderode, Christian Dietrich Grabbe, Friedrich Hölderlin, Heinrich von Kleist ou Jakob Michael Reinhold Lenz, car elle y vit des parallèles avec sa propre expérience. L'aliénation, l'absence de perspective en dehors des voies toutes tracées auxquelles on ne pouvait échapper et qui ne laissaient pas de place à la personnalité individuelle, le sentiment d'agir dans le vide et de tourner en rond, le ressenti schizophrène du gouffre entre idéal et réalité, tous ces sentiments étaient aussi forts que dans la première moitié du XIX ${ }^{\mathrm{e}}$ siècle $^{24}$. travail à une discussion sur Dantons Tod. En octobre 1977, le célèbre Prix Büchner (RFA) fut remis à l'écrivain transfuge Reiner Kunze qui venait de quitter la RDA. La même année, Volker Braun écrivit un essai intitulé «Büchners Briefe ${ }^{25}$ qui établissait un parallèle ostensible entre la déception du révolutionnaire hessois et celle des intellectuels est-allemands. En 1978, à la Volksbühne de Berlin, Jürgen Gosch osa une mise en scène audacieuse de Leonce und Lena qui fut suspendue au bout de quelques représentations. Dans un tel contexte, à la seule annonce d'opéras d'après Büchner, on pouvait s'attendre à des opéras fortement politisés. Or, tant Dessau que Hertel choisirent précisément la pièce de Büchner en apparence la moins politique qui fût, ce qui n'excluait cependant pas un traitement politique du matériau théâtral.

\section{Structure dramatique des opéras}

Les deux opéras ont pour point commun d'être des opéras de chambre tant par leur durée (un peu plus d'une heure) que par l'effectif vocal et orchestral (quinze musiciens dans l'opéra de Hertel $^{26}$ et une petite trentaine dans l'opéra de Dessau mais avec un traitement soliste de chaque instrument ${ }^{27}$ ). Dessau ne voulait pas composer de long opéra comme il en avait coutume, car il savait que son temps était compté. Quant à Hertel, on peut supposer que l'innovation que représentait pour lui la composition d'un opéra le fit s'en tenir à des proportions modestes. Il souhaitait que son opéra soit « intime » et qu'il n'y ait pas de fosse pour l'orchestre ${ }^{28}$.

Malgré ces proportions comparables, les deux opéras se distinguent radicalement par la construction de leurs livrets. Le livret est de Thomas Hertel et Karla Kochta («dramaturge » au Staatstheater de Dresde) pour le premier et de Thomas Körner pour Dessau. Körner avait déjà réalisé le livret de Goldmann R. Hot. Aussi Dessau savait-il à qui il s'adressait même si Körner était peu connu. Körner prit un parti extrême qui fut critiqué, mais qui était un choix esthétique auquel Dessau adhérait : il mit la pièce de Büchner sens dessus dessous et prit l'intrigue à rebours. Körner se désignait lui-même non comme "librettiste», mais comme "organisateur de texte». La construction d'ensemble de l'opéra est tout à fait asymétrique : le deuxième acte ne dure que quinze minutes, contre environ quarante minutes pour le premier. Les trois actes de la comédie de Büchner - exposition, péripétie, dénouement - se voient réduits à deux.

Hertel et Kochta, moins audacieux, en restèrent à l'ordre des scènes de la pièce d'origine, renonçant cependant à la division en actes et juxtaposant dix « tableaux ».

Avant d'indiquer la structure dramatique de chacun des opéras, précisons que dans les deux cas, toutes les répliques étaient de la plume même de Büchner. Mais tandis que Hertel et sa librettiste reprirent le texte original à la lettre en se contentant d'y faire des coupures, Körner ne reprit que des bribes des phrases (voire de mots) de Büchner, 
laissant ces bribes telles quelles, à l'état brut, sans chercher (en apparence) à les ordonner.

Leonce und Lena de Hertel ${ }^{29}$ :

Pas d'ouverture

1. Bild (I,1) ${ }^{30}$ Leonce (Prince) et Valerio (son serviteur) mènent un court dialogue absurde, puis monologue de Leonce sur l'ennui et l'oisiveté. Retour de Valerio, dialogue absurde, chant «Fleig an der Wand» («mouch" sur le mur»), éloge de la folie.

2. Bild (I,2) Le Roi (marionnette) est habillé et tient des propos pseudophilosophiques (sur trois notes seulement). Conseil d'État.

3. Bild (I,3) Leonce et Rosetta (mime). Elle danse, Leonce la renvoie. Monologue de Leonce sur les femmes et le vin. Le Président et le Conseil d'État (muets) apportent une lettre à Leonce, qui y apprend qu'il doit se marier. Valerio arrive, se moque de Leonce. Ne voulant pas être des membres utiles de la société, ils décident de partir en Italie.

4. Bild (I,4) Nature. Lena chante pour exprimer qu'elle préfère la mort au mariage. Gouvernante (mime) l'écoute.

5. Bild (II,1-2) Sur le chemin de l'Italie. Valerio se plaint du chemin. Leonce rêve d'«idéaux » et notamment d'une " dame très belle et sans esprit ». Valerio boit. Leonce se plaint d'être si jeune alors que le monde est si vieux. Ils recherchent une activité. Monologue lyrique de Leonce sur la beauté de la nature. Apparition de Lena et de la gouvernante. Leonce et Lena se rencontrent et partagent les mêmes pensées sur la longueur du chemin (Lena : «Le chemin est-il si long?» - Leonce : "Oh, tous les chemins sont longs »). Valerio en conclut : « il est fou ».

6. Bild (II,3) Sur un balcon à la nuit tombante. Monologue de Lena qui s'adresse à sa gouvernante et parle de Leonce avec exaltation.

7. Bild (II,4) Trio: Valerio se plaint des moustiques, Leonce et Lena admirent la nature. Duo de Leonce et Lena autour du thème du rêve. Leonce embrasse Lena. Elle s'enfuit. Leonce veut se suicider, mais Valerio l'en dissuade.

8. Bild (III,1) Plaine, le jour. Leonce annonce à Valerio son intention de se marier. Valerio, étonné, demande s'ils connaissent l'identité l'un de l'autre. Réponse négative. Valerio accepte néanmoins d'aider Leonce à se marier s'il est fait ministre. Pacte conclu.

9. Bild (III,2) Orchestre se lève et annonce aux paysans « en canon » que le couple royal arrivera le lendemain et qu'il faudra bien se tenir. Orchestre termine sur « vivat » mais pas de réponse des paysans (car ce sont des marionnettes).

10. Bild (III,3) Tout le monde (orchestre et le Roi) crie "vivat » avec amplification électroacoustique. Le Roi rappelle sa décision de se réjouir et décide de la mettre en œuvre même en l'absence de son fils. Leonce, Lena et Valerio arrivent masqués. Valerio présente Leonce et Lena comme des automates et propose de les marier. Mariage puis coup de théâtre : Leonce et Lena ont été mariés l'un à l'autre sans savoir qu'il s'agissait de celui/celle qui leur était prédestiné par le Roi. Le Roi remet le pouvoir aux mains de son fils. Leonce, Lena et Valerio seuls. Leonce constate qu'ils ont les "poches pleines de poupées", veut arrêter le temps et mettre des miroirs dans tout le pays. Valerio veut interdire le travail. Tous trois finissent en fredonnant.

Leonce und Lena de DESSAU :

Einleitungsmusik puis Nachspiel

Vorspiel (III,2) On prépare les paysans au passage du couple royal (Leonce: «E la fama ?»-Paysans : «Vi ! »-Valerio : «E la fame ${ }^{31}$ - Paysans : «Vat ! )

I, 1 (III,1) Leonce annonce à Valerio son intention de se marier avec une femme dont il ne connaît pas le nom, Valerio sera ministre s'il l'aide.

I, 2 (II,4) Nuit au clair de lune. Valerio peste contre la nature. Leonce et Lena se rencontrent autour du thème de la mort et du rêve. Baiser, puis Lena s'enfuit. Suicide raté de Leonce, fierté de Valerio.

I, 3 (II,3) La gouvernante recommande à Lena d'oublier Leonce. 
I, 4 (II,2) Sur la route : Valerio donne à boire à Leonce. Leonce se plaint d'être jeune alors que le monde est vieux. Ils rencontrent Lena et sa gouvernante. Dispute Valerio-gouvernante, tandis que les pensées de Leonce et Lena se rencontrent.

I, 5 (II,1 inversé) Sur le chemin : d'abord Lena et gouvernante qui se plaignent de la nature. Puis Valerio et Leonce: Valerio se plaint. Leonce fait le portrait de la femme "idéale ", puis à nouveau plainte de Valerio. Se conclut sur une rêverie de Lena (scène en boucle)

I, 6 Enchaînement de « mini-scènes » :

- $(I, 4)$ Lena pleure sur son sort, la gouvernante a une idée.

- (I,3 a) Leonce et Rosetta (elle danse, mais il la renvoie)

- (I,2 inversé) D'abord le Conseil d'État, puis le roi est déshabillé (et non habillé)

- (I,3 b) Leonce seul, réflexion sur l'amour, puis Valerio. Le président vient annoncer l'arrivée de Lena pour le lendemain. Valerio et Leonce cherchent que devenir et lancent des mots en l'air. Leonce refuse d'être un membre utile à la société.

II, 1 (III, 3) Jour de la cérémonie : au début, désolation, personne ne trouve Leonce et Lena. Le Roi veut se réjouir. Valerio arrive masqué, présente les automates, le Roi les marie en effigie. Coup de théâtre : révélation des identités. Leonce remercie et congédie les serviteurs, les informant qu'ils recommenceront la plaisanterie depuis le début le lendemain [effectivement, la dernière scène est la $1^{\text {ère }}$ scène chez Büchner !] Rêve d'un paradis terrestre.

II, 2 (I,1) Leonce et le précepteur, puis arrivée de Valerio. Valerio et Leonce mettent un doigt sur le nez (folie) puis chantent «Fleig' an der Wand» («Mouch' sur le mur »). Leonce : «Wär ich doch ein Narr ! Mein Ehrgeiz geht auf eine bunte Jacke » ( "Oh si je pouvais être un bouffon! Un habit bariolé, voilà mon ambition » : citation de Shakespeare placée par Büchner en épigraphe). Valerio et Leonce font répéter le Vivat au chœur et intervertissent leurs interrogations du début (Leonce « $\mathrm{E}$ la fame ? », Valerio : « E la fama? ». Le chœur répond « Vat ! Vi ? »).

21 Le livret de l'opéra de Hertel reprend l'ordre de la pièce originale tout en effectuant quelques coupures dans le texte. Le seul ajout notoire que le livret introduit est la mention «black » entre la plupart des scènes : l'absence volontaire de transition d'une scène à l'autre, déjà présente chez Büchner, est ici mise en exergue. Cependant, la simple copie du texte büchnérien amputé de certains passages peut laisser craindre que la fidélité à la lettre du texte n'aille pas de pair avec la fidélité à l'esprit büchnérien. La comédie de Büchner, en effet, par son second degré permanent, son intertextualité, ses pastiches et collages ne peut justement être prise « au pied de la lettre ». Nous verrons que dans l'opéra de Hertel, l'ironie est retranscrite non dans le livret mais dans la musique, par l'utilisation incessante de citations.

Dans l'opéra de Dessau, en revanche, le jeu de pastiche et de collage a lieu dès l'élaboration du livret : Körner joue avec le texte de Büchner, met la pièce sens dessus dessous, restant ainsi moins fidèle à la lettre du texte original qu'à son esprit, caractérisé par le jeu avec le matériau littéraire, le collage, la fragmentation. Le principe organisateur de l'inversion - placé sous le signe d'une phrase liminaire de Leonce « wer sich einmal auf den Kopf sehen könnte !» («Oh, voir une fois le dessus de sa tête!", I,1 chez Büchner) - est appliqué à toutes les échelles, y compris à celle des mots, si bien que les syllabes de certains mots sont inversées, ou que la logique des répliques d'un dialogue n'est parfois plus reconstituable. Ainsi nombre de phrases de Büchner sont retournées en leur contraire par la simple élision de la négation (I,4 : «Damit Sie, Geehrteste, sich [nicht] die Nase am Horizont blutig stoßen ». I, 5 : «Die Blumen sind ja [kaum] welk ») ou par une discrète substitution de mot (II,1 : « das war die Flucht aus [ins] dem Paradies »). 
23 L'ouverture de l'opéra de Dessau est révélatrice: elle est composée d'une « introduction » instrumentale, suivie d'un court « postlude » (instrumental lui aussi), sur lequel se greffe ensuite un "prélude » radicalement différent et chanté. L'ordre logique est bouleversé puisque le postlude précède le prélude. L'auditeur s'en aperçoit au plus tard quand le prélude est repris à la fin de l'opéra avec des paroles inversées ( "Vat-vi » au lieu de «Vi-vat») et interverties entre Leonce et Valerio (« E la fame ? E la fama?»). Le texte du prélude lui-même est par ailleurs ostensiblement construit en boucle (le «vivat» des paysans l'ouvre et le ferme). On a donc plusieurs indications pour la suite de l'opéra : la construction en boucle, le thème annoncé de la dialectique de la faim (fame) et de la réputation (fama) et enfin, la fragmentation tant du discours musical (cellules juxtaposées) que textuel (scission syllabique « vi-vat »).

24 À la fin de l'opéra, Dessau et Körner jouent sur la dialectique de l'ouverture et de la fermeture, mettant d'un côté en exergue la construction en boucle de l'opéra par le retour du prélude et la réapparition des paysans, mais achevant d'un autre côté leur œuvre sur un point d'interrogation: l'acclamation initiale est retournée en questionnement: "Vat! Vi ?», ce qui signifie en dialecte hambourgeois (Dessau était originaire de Hambourg) «was! wie?» (« quoi! comment?»). En ouvrant la fin de l'opéra par un point d'interrogation (qui exprime l'indignation des paysans face au statu quo de la société), Dessau et Körner restaient fidèles à la comédie büchnérienne : en effet, si la forme de cette dernière était fermée en apparence, ce n'était que pour mieux condamner l'artificialité du modèle de la comédie classique. Büchner dénonçait en l'exagérant le point de vue téléologique qui préside implicitement à une action dramatique bien ficelée. La tension dramatique de la pièce de Büchner n'était que feinte et les ressorts traditionnels de la comédie (coups de théâtre à répétition) étaient mis à dessein en exergue. Dessau et Körner vont plus loin dans la déconstruction du modèle classique car ils détruisent toute tension dramatique en présentant les scènes dans un ordre qui semble aléatoire.

Dans l'opéra de Hertel, l'ironie s'exprime différemment : la musique mine ironiquement les crescendi dramatiques. Ainsi la scène du baiser, l'un des points culminants de la pièce de Büchner, frappe par l'absence d'harmonie entre les deux amoureux. Alors que la scène commence par un trio dans lequel les voix de Leonce et Lena se répondent et semblent évoluer vers un duo, dès que Valerio arrête de chanter, Leonce et Lena s'expriment en un parlé-chanté (Sprechgesang) beaucoup moins fusionnel ; chacun a une ligne vocale indépendante de l'autre et au lieu de se répondre, les voix se superposent. Tout se passe comme si le compositeur voulait éviter le procédé rebattu à l'opéra qui consiste à faire culminer les scènes d'amour en des duos fusionnels.

D'autres coups de théâtre font l'objet d'une dramatisation musicale qui repose sur l'excès : ainsi en va-t-il de la tentative de suicide de Leonce, qui, par son exagération, ne saurait impliquer sentimentalement l'auditeur, mais le maintient au contraire à distance, ou encore de la "frénésie » de Leonce et Valerio au début, dont on perçoit aisément qu'il ne s'agit que d'un jeu auquel les deux « héros » eux-mêmes ne croient pas.

27 Les effets de rupture entre texte et musique sont mis en exergue chez Hertel par l'insertion incessante de citations musicales très célèbres. Le public, qu'il eût une culture musicale ou non, ne pouvait que percevoir l'humour sous-jacent. L'écriture de Hertel est en effet sérielle, tandis que les citations, non retravaillées (mis à part dans leur instrumentation), sont tonales et tranchent sur le reste du discours musical. Les 
citations musicales sont la plupart du temps un commentaire ironique de l'action. Ainsi en va-t-il à la fin de la première scène de la citation du choral des hommes armés de la Flûte Enchantée : dans l'opéra de Hertel, il n'y aura en réalité pas d'épreuve pour les deux «héros " puisque ces derniers reviennent dans leur pays d'origine juste après l'avoir quitté. La fanfaronnerie de Valerio ("trom-trom-pläre », sc. 3) est pour sa part illustrée par la Marche militaire de Schubert: Valerio apparaît là encore sous un jour ironique. Les scènes entre Leonce et Valerio sont truffées de citations dont la fonction semble être d'illustrer la divagation des personnages. La rupture a lieu au sein du tissu musical lui-même qui se présente comme un «habit d'Arlequin» et met ainsi en application la citation de Shakespeare que Büchner avait placée en épigraphe de sa comédie (« Wär ich doch ein Narr! Mein Ehrgeiz geht auf eine bunte Jacke »).

Le tissu musical de l'opéra de Dessau repose lui aussi sur une juxtaposition de fragments, mais à bien plus petite échelle : les cellules musicales sont de quelques notes seulement. Dessau ne fait pas usage de citations dans cet opéra (si l'on excepte les mélodies d'inspiration hébraïque de Lena et Rosetta). On ne reconnaît pas le style musical de Dessau, lui que les musiciens surnommaient «Paukenpauli $»^{32}$, car la musique est très confidentielle, les instruments sont traités en solistes, les cordes divisées. Les relais de timbres et la juxtaposition de cellules musicales extrêmement courtes font penser au style d'Anton Webern. Malgré la discrétion de l'accompagnement instrumental, les paroles sont difficilement compréhensibles car les lignes vocales sont extrêmement éclatées. Les effets de fragmentation des mots viennent se joindre à l'éclatement de l'ambitus. Ce traitement de la voix allié à la décision du librettiste de retourner l'action fait de cet opéra une œuvre difficile à comprendre, voire incompréhensible à la première écoute.

La création posthume suscita beaucoup d'étonnement chez un public que Dessau avait habitué à des messages plus clairs, d'autant que la mise en scène de Ruth Berghaus obscurcissait le sens de l'opéra. Berghaus prit par exemple le parti de faire rester les paysans sur scène d'un bout à l'autre de l'opéra, alors que ces derniers ne chantaient qu'au tout début et à la toute fin. Selon les témoignages de l'époque, cet élément de mise en scène qui n'était indiqué ni dans le livret ni dans la partition perturbait la compréhension de l'action dramatique représentée au premier plan et ajoutait donc une strate de signification que les spectateurs étaient libres d'interpréter à leur guise. La présence scénique constante des paysans pouvait par exemple souligner l'indifférence des dirigeants au sort des pauvres gens : si les questions de la faim et de la réputation n'étaient toujours pas résolues à la fin de l'opéra, c'était par la faute du prince Leonce et de la princesse Lena, qui, après avoir tenté la fuite, se satisfaisaient finalement de leur Tour d'ivoire et se réfugiaient dans une utopie régressive. Selon cette hypothès ${ }^{33}$, Ruth Berghaus aurait cherché à mettre en exergue le thème inscrit au cœur de la déstructuration dramatique de l'opéra de Dessau : la remise en question de l'utopie.

\section{Potentiel subversif des opéras d'après Büchner en RDA}

30 Le Royaume idyllique où l'on n'a pas à gagner son pain à la sueur de son front décrit conjointement par Leonce, Lena et Valerio à la fin des opéras ${ }^{34}$ est présenté comme sclérosé, refermé sur lui-même, indifférent à l'extérieur. Sans doute cette dénonciation 
était-elle à lire à un double niveau : dénonciation de l'utopie dont la RDA avait prétendu être la réalisation et à laquelle elle aspirait toujours. D'autre part, de même que Büchner parodiait la comédie classique, tant Dessau que Hertel prenaient leurs distances avec une conception utopiste de l'opéra tel que l'imaginaient les instances culturelles de la RDA.

31 Les principes aristotéliciens déjà remis en cause par Büchner l'étaient encore plus par Hertel et Dessau : Hertel multipliait les citations musicales anachroniques superposant ainsi dans son opéra des strates temporelles qui faisaient éclater l'unité de temps et de lieu; Dessau quant à lui faisait voler en éclats les unités aristotéliciennes en fragmentant le texte à outrance et en réorganisant les scènes dans un ordre quasialéatoire. Cette déconstruction sapait la vision téléologique de l'Histoire que «l'opéramodèle» de RDA se devait de véhiculer et prenait le contre-pied de «l'héritage classique et humaniste » tant célébré en RDA.

Les compositeurs cherchaient visiblement à gommer les repères d'écoute : dans l'opéra de Dessau, la déstructuration de la trame dramatique et du langage même s'apparente à l'esthétique du théâtre postdramatique qui « détrône la synthèse » pour privilégier une structure éclatée, parataxique, non-linéaire, proche de celle du rêve et qui invite le spectateur à se mettre en quête d'un sens caché3 ${ }^{35}$; sans aller aussi loin, les collages incessants dans la musique de Hertel perturbaient l'écoute dans la mesure où ils suggéraient un deuxième degré de signification. Le procédé de juxtaposition ou superposition d'éléments à première vue totalement étrangers les uns aux autres pourrait être qualifié d'" hétérotopie " pour reprendre la distinction que fait Foucault entre l'hétérotopie qui inquiète et l'utopie qui rassure ${ }^{36}$. La forme ouverte de l'œuvre (par la fragmentation chez Dessau et l'intertexte chez Hertel) et l'absence de tout point de vue directeur invitent le public à prendre un recul critique face à l'idylle de Leonce et Lena. La mise à distance permanente du monde figé dénonce ainsi la pétrification de l'utopie. Florence Baillet ${ }^{37}$ parle en ce sens (et au sujet du théâtre allemand précédant immédiatement la chute du Mur de Berlin) de «dystopie » : afin d'éviter le piège de la pétrification idéologique, la « dystopie » emprunte des voies retorses, notamment celle de la négation. Cela se traduit dans l'opéra de Dessau, et à moindre échelle dans celui de Hertel, par l'absence de tout point de vue surplombant. Rien n'est affirmé avec autorité : Hertel ne cesse de se distancer ironiquement par la musique de ce qui est dit sur scène, Dessau démontre que toute proposition peut être retournée imperceptiblement en son contraire et atomise les mots au point de les vider de leur sens (scène 1 de l'acte II : « Ein königliches Wort ist ein Ding, Ding, Ding, Ding, dingding, no-thing »).

Comme le dit Florence Baillet au sujet de Hamlet-Machine de H. Müller, « on est loin du texte lisse ordonné autour d'une fable, tel que le prône le réalisme socialiste : il offre des trous, des lacunes, afin d'être aussi 'l'affaire du public' qui est libre de donner ou non un sens aux énigmes de ce sphinx $»^{38}$. La déconstruction de toutes les certitudes n'est-elle pas ce qui offre la possibilité de surgissement de nouveauté ? Le « vat ? vi ! » à la fin de l'opéra de Dessau et l'idée büchnérienne de reprendre tout depuis le début ("Morgen fangen wir in aller Ruhe den Spaß noch einmal von vorne an ») ne sont-ils pas porteurs d'espoir, au-delà du cynisme qui y est immédiatement perceptible? artistes de RDA dans les années 1970 et $1980^{39}$. Aussi nous semble-t-il justifié de recourir ici à Walter Benjamin pour décrire le « vat? vi ! final des paysans chez Dessau : cette 
onomatopée marque un réveil soudain des paysans, un arrachement hors du rêve. L'utopie décrite juste avant par Valerio, Leonce et Lena d'un pays où l'on n'a pas à travailler, rêve collectif s'il en est, est remise en question par l'onomatopée interrogative "Vat? vi!». Ce brusque réveil coïncide précisément avec la fin de l'opéra, lieu de l'u-topie par excellence. On peut peut-être assimiler l'onomatopée finale à un " arrêt sur image » benjaminien, défini par Miguel Abensour comme le moment où "la contradiction explose, libérant une virtualité émancipatrice » ${ }^{40}$ et qui "sauve l'utopie en s'efforçant de l'arracher au mythe afin de la transformer en image dialectique $»^{41}$. Seul ce réveil, cet arrachement hors du rêve ouvre la possibilité de surgissement de nouveau.

C'est ainsi que la pièce en apparence la moins politique de Büchner fut l'objet d'un traitement politique à l'opéra. En effet, comme le dit Hans-Thies Lehmann au sujet du théâtre postdramatique, « ce n'est pas par la thématisation directe du politique que le théâtre devient politique mais par la signification implicite de son mode de représentation $»^{42}$.

\section{Conclusion}

L'opéra en RDA avait pour spécificité d'être en lien direct avec la vie de la Cité. Aussi n'est-ce pas « sur-interpréter » les opéras d'après Büchner que de «traiter ce[s] œuvres comme un signe intentionnel hanté par quelque chose d'autre, dont elle[s] [sont] aussi symptôme $»^{43}$. Le public est-allemand n'était pas sourd à l'actualité de Büchner : lorsque Leonce und Lena fut mis en scène par Jürgen Gosch à la Volksbühne en 1978, des discussions avec le public ${ }^{44}$ révélèrent que beaucoup voyaient des parallèles entre la sclérose des royaumes «Pipi» et «Popo» et la paralysie de la RDA. Cette représentation théâtrale fut bientôt suspendue et il semblerait que l'opéra ait pris le relais et ait bénéficié d'une certaine marge de liberté que n'avait pas le théâtre.

La comédie Leonce und Lena de Büchner reposait sur un jeu avec les formes de la comédie, avec ses conventions, son répertoire quasi figé et immuable. Cette comédie permettait une souplesse d'adaptation, réclamait même une poursuite du jeu de déformation et de recyclage de matériau littéraire entrepris par Büchner. Aussi l'adaptation de cette comédie à l'opéra obligea-t-elle les compositeurs, librettistes et metteurs en scène à se montrer inventifs, à renouveler les formes de l'opéra tout en jouant avec des formes préexistantes. Dessau, en ôtant à la pièce presque toute logique dramatique et en déconstruisant le langage même, alla très loin dans la subversion de l'idéal classique, transformant la comédie parodique de Büchner en opéra postdramatique.

Quant à l'opéra de Hertel, s'il conservait la trame dramatique de la comédie d'origine, son "hétérotopie » musicale fort déroutante fit dire à un critique musical de l'époque que les créateurs avaient "réussi à faire transparaître tant la poésie que le regard critique de Büchner sur ses personnages. Cet opéra n'est pas seulement beau, mais a été composé pour inciter les auditeurs à la réflexion $»^{45}$. Pour revenir à Bourdieu, il faut en effet souligner que «mettre en forme, c'est aussi mettre les formes, et [que] la dénégation qu'opère l'expression littéraire est ce qui permet la manifestation limitée d'une vérité qui, dite autrement, serait insupportable $»^{46}$ 


\section{NOTES}

1. Georg Lukács : «Der faschisierte und der wirkliche Georg Büchner » in : Das Wort, 19.02.1937, 2/2, p. 7-26.

2. En 1955 pour la réouverture de la salle (dans un étonnant sursaut d'ouverture à la modernité au milieu de la lutte contre le « formalisme »), puis seulement à nouveau en 1984 et 1987.

3. CD chez Berlin Classics (1074-2) : Staatskapelle de Berlin, dir. Otmar Suitner. Réduction piano disponible chez Alkor Edition (Henschel). Partition d'orchestre aux archives Dessau, Stiftung Archiv der Akademie der Künste (SAdAK), Berlin.

4. Enregistrement disponible au Deutsches Rundfunkarchiv (Babelsberg). Partition originale conservée à la Sächsische Landesbibliothek - Staats- und Universitätsbibliothek Dresden.

5. Entretien personnel le 24 juillet 2006 à Leipzig.

6. Pierre Bourdieu: Les Règles de l'art. Genèse et structure du champ littéraire, Paris, Seuil, 1998 $\left(1992^{1}\right)$, Points Essais n³63, p. 150.

7. Ibid., p. 177.

8. Lettre de Boris Blacher à Gottfried von Einem : Dein Projekt mit Leonce und Lena halte ich nicht für eine glorreiche Idee. Der Stoff ist hübsch, aber zu sehr an das Wort gebunden, um als Oper zu wirken». Stephan Mösch : Der gebrauchte Text. Studien zu den Libretti Boris Blachers, Stuttgart, Metzler, 2002, p. 83.

9. Voir notamment l'appel à un «opéra national » en première page du Neues Deutschland du $1^{\text {er }}$ novembre 1952.

10. Opéras de Paul Dessau notamment.

11. R. Hanell : Die Spieldose (1957), Dorian Gray (1962), Esther (1966), Griechische Hochzeit (1969). O. Gerster : Der fröhliche Sünder (1963).

12. Old Fritz de Kunad (1963), Lazarillo von Thormes de Matthus (1964), Die Passion des Johannes Hörder de J.-K. Forest (1965), Die weiße Rose de Udo Zimmermann (1967). Voir Sigrid et Hermann Neef : Deutsche Oper im 20. Jahrhundert, DDR, 1949-89, Berlin, Peter Lang, 1992.

13. Certains comme E. H. Meyer (Reiter der Nacht en 1973) ou R. Hanell (Babettes grüner Schmetterling en 1982) continuèrent néanmoins à composer dans un style néoclassique.

14. Contrairement à Eisler notamment, qui garda la nationalité autrichienne.

15. Lettre du 30.12.1976 de Hans Pischner, Directeur du Staatsoper à Dessau, in : «Briefe Staatsoper Berlin an Paul Dessau », Stiftung Archiv der Akademie der Künste (SAdAK), Paul Dessau Archiv (PDA), cote 2024.

16. Daniela Reinhold (éd.) : Paul Dessau 1894-1979. Dokumente zu Leben und Werk, Berlin, Henschel, 1995 , p. 88.

17. Journal de Dessau daté de septembre 1978, in : Daniela Reinhold (éd) : Paul Dessau 'Let's hope for the best' : Briefe und Notizbücher aus den Jahren 1948 bis 1978, Berlin, Wolke, 2000, p. 285.

18. Quelques mois plus tard, l'opéra fut également joué en RFA, à Fribourg-en-Brisgau, dans une mise en scène de H.-L. Kindermann. Contrairement aux autres œuvres de Dessau, cet opéra ne fut pas repris par la suite.

19. Par exemple «Dokumentation » für Grafiken (H. H. Grimmling) und 14 Instrumente (1974).

20. Il avait fait ses dernières recommandations concernant ses obsèques, refusant que ces dernières soient des obsèques nationales.

21. Entretien personnel (2006).

22. Témoignage de Maxim Dessau, fils du compositeur, au cours d'un entretien personnel en septembre 2006 à Berlin.

23. Son drame Dantons Tod ne fut pas mis en scène avant 1963 par Kurt Barthel à Rostock, dans une version où certaines scènes avaient été réécrites pour en simplifier le message idéologique. 
On attendit ensuite dix ans pour rejouer Dantons Tod en RDA en 1973 à Magdebourg. Voir Ulrich Kaufmann : Dichter in "stehender Zeit »: Studien zur Georg-Büchner-Rezeption in der DDR, Erlangen, Palm und Enke, 1992. Dietmar Goltschnigg (éd) : Georg Büchner und die Moderne. Texte, Analysen, Kommentar, vol. 2 (1945-1980) et 3 (1980-2000), Berlin, Erich Schmidt, 2002, 2004.

24. Daniela Reinhold : Paul Dessau. Dokumente zu Leben und Werk, op.cit., p. 88 (traduction : L. D.).

25. Publié immédiatement en France dans la revue Connaissance de la RDA, 1978, $\mathrm{n}^{\circ} 7$ (octobre), p. 8-17.

26. Quatre bois (flûte, hautbois, clarinette, saxophone), deux cuivres (trompette, trombone), trois instruments à cordes frottées (violon, alto, violoncelle), piano et guitare électriques, guitare basse, dispositif électroacoustique et nombreuses percussions réparties entre trois instrumentistes (Glockenspiel, xylophone, guimbarde, woodblocks, tambour, cymbales, cloches, marimba etc.)

27. Chez Dessau, la nomenclature des vents parait assez traditionnelle, avec des bois par deux, des cuivres par trois (trompettes, trombones) ou quatre (cors). On remarque une grande importance des percussions à hauteur déterminée (celesta, marimba, Glockenspiel, timbales), la présence d'instruments à cordes frappées (piano) et à cordes pincées (harpe). La nomenclature des cordes est plus étonnante: les violons, peu nombreux (huit), ne sont pas regroupés par pupitre, mais ont chacun leur propre partie. Il en va de même pour les trois altos, le violoncelle et les deux contrebasses.

28. Émission radiophonique «Musikklub» en 1981 : «Leonce und Lena zur Diskussion », SAdAK, AVM, 32.8765 et 32.8766 .

29. Sous-titre : « Oper in 10 Bildern für Schauspieler, Sänger, Tänzer und Marionetten nach dem gleichnamigen Lustspiel von Georg Büchner». Le Roi, Leonce et Lena sont des acteurs (qui chantent). Seul Valerio est interprété par un chanteur, la gouvernante et Rosetta par des danseuses. Le Président, le conseil d'État et de la Cour ainsi que les paysans sont des marionnettes.

30. Les chiffres entre parenthèses indiquent la scène correspondante dans la pièce de Büchner.

31. En latin, fama: la réputation, l'opinion publique. Fame (de fames) : la faim. Il s'agit d'une reprise du court avant-propos de Büchner qui fait lui-même référence à Alfieri pour le genre tragique et à Gozzi pour le genre comique : «Alfieri : 'E la fama ?' Gozzi:'E la fame?' ».

32. Jeu de mot sur Pauken (timbales) et « Pauli », diminutif de Paul (prénom de Dessau).

33. Hypothèse confirmée par des documents de conception de mise en scène retrouvés dans les archives de Ruth Berghaus (SAdAK).

34. Chez Dessau : Leonce : «Wollen wir ein Theater bauen? Lena : Besser alle Uhren zerschlagen, Kalender verbieten (...) und dann umstellen wir das Ländchen mit Brennspiegeln, daß es keinen Winter mehr gibt. Das ganze Jahr zwischen Rosen und Veilchen, zwischen Orangen und Lorbeer Valerio : Wer sich krank arbeitet ist strafbar ».

35. Hans-Thies Lehmann : Postdramatisches Theater, Frankfurt am Main, Verlag der Autoren, 2001. 36. Michel Foucault : Les mots et les choses. Une archéologie des sciences humaines, Paris, Gallimard, 1996.

37. Florence Baillet : L'utopie en jeu : critiques de l'utopie dans le théâtre allemand contemporain, Paris, CNRS Editions, 2003. Florence Baillet emprunte le terme de «dystopie » à Dragan Klaic et JanRobert Bloch.

38. Ibid., p. 99.

39. Wolfgang Bialas : «Zur Rezeption der Frankfurter Schule in der DDR » in : Deutsche Zeitschrift für Philosophie, 1995, 43/1, p. 131-142.

40. Miguel Abensour : L'Utopie de Thomas More à Walter Benjamin, Paris, Sens \& Tonka, 2000, p. 202.

41. Ibid., p. 21.

42. Hans-Thies Lehmann : Postdramatisches Theater, Frankfurt am Main, Verlag der Autoren, 2001, p. 279. 
43. Bourdieu : op. cit., p. 16.

44. Protocoles des discussions aux archives de l'Akademie der Künste.

45. Émission radiophonique « Musikklub», op. cit.

46. Bourdieu : op. cit., p. 69.

\section{RÉSUMÉS}

Cet article compare deux traitements opératiques d'un même matériau théâtral (Leonce und Lena de Büchner) dans une perspective esthético-historique : celle de la RDA au tournant des années 1970-80.

Nous retraçons d'abord les circonstances de création des opéras Leonce und Lena de Paul Dessau (1979) et de Thomas Hertel (1981) en rappelant la place particulière accordée à l'opéra en RDA et l'actualité brûlante de Büchner dans les années 70 après deux décennies de quasi-censure.

La description des livrets et de l'interaction des trois arts en présence permet de mettre en évidence la singularité des choix esthétiques de chacun des opéras. Malgré toutes les différences d'agencement et de style musical qui les séparent, les deux opéras se rejoignent dans leur volonté d'individualiser musique, texte et scène. Dans l'opéra de Hertel, la musique prend ainsi souvent une distance ironique à l'égard du texte par l'usage de pastiches musicaux ou de citations plus sérieuses. Dessau et son librettiste font usage d'une esthétique du fragment très déroutante qui détruit la syntaxe et fait surgir l'étonnement.

Ni Dessau ni Hertel ne cherchent à rassurer l'auditeur par une fable bien ordonnée, à faire tenir ensemble les mots et les choses: la négation de l'utopie, tant celle prônée à l'opéra par les autorités politico-culturelles, que celle qu'était censée incarner la RDA, est au centre des deux œuvres.

Dieser Aufsatz vergleicht in einer ästhetisch-geschichtlichen Hinsicht zwei um 1980 in der DDR entstandenen Veroperungen des gleichen Theaterstücks (Leonce und Lena von Büchner).

Zuerst werden die Umstände beschrieben, unter denen die Opern Leonce und Lena von Paul Dessau (1979) und Thomas Hertel (1981) uraufgeführt wurden. Die besondere Rolle, die der Oper in der DDR zugeschrieben wurde, und die brisante Akualität Büchners nach zwei Jahrzehnten praktischer „Zensur“ werden dabei unterstrichen.

Die Beschreibung der Libretti und des Zusammenspiels der drei operinnewohnenden Künste führt dazu, die ästhetischen Besonderheiten jeder Oper hervorzuheben. Trotz aller Aufbau- und Stilunterschiede streben beide Opern nach der Autonomie jeweils der Musik, des Textes und der Bühne: In Hertels Oper nimmt die Musik oft Distanz zum Text durch ironische Nachahmungen oder ernst gemeinte Zitate ein. Dessau und sein Librettist verwenden eine verwirrende Ästhetik der Fragmentation, die die Syntax zerstört, und beim Publikum Erstaunen auslöst.

Weder Dessau noch Hertel möchten dem Hörer durch eine schön aufgebaute Fabel entgegenkommen, Ausdruck und Bedeutung zusammenhalten: Die Negation der Utopie, sowohl der, die die Oper nach Wunsch der ostdeutschen kulturellen Behörden darstellen sollte, als auch der, die die DDR angeblich verkörperte, steht im Mittelpunkt beider Opern. 
INDEX

Mots-clés : RDA, utopie

oeuvrecitee Leonce und Lena

\section{AUTEURS}

\section{LAETITIA DEVOS}

Université François Rabelais, Tours 